\title{
Positivity of Amplitudes, Weak Gravity Conjecture, and Modified Gravity
}

\author{
Brando Bellazzini® and Matthew Lewandowskiఠ \\ Institut de Physique Théorique, Université Paris-Saclay, CEA, CNRS, F-91191 Gif-sur-Yvette, France \\ Javi Serra๑ \\ Physik-Department, Technische Universität München, 85748 Garching, Germany
}

(Received 23 September 2019; published 18 December 2019)

\begin{abstract}
We derive new positivity bounds for scattering amplitudes in theories with a massless graviton in the spectrum in four spacetime dimensions, of relevance for the weak gravity conjecture and modified gravity theories. The bounds imply that extremal black holes are self-repulsive, $M /|Q|<1$ in suitable units, and that they are unstable to decay to smaller extremal black holes, providing an $S$-matrix proof of the weak gravity conjecture. We also present other applications of our bounds to the effective field theory of weakly broken Galileons, axions, and $P(X)$ theories.
\end{abstract}

DOI: 10.1103/PhysRevLett.123.251103

Introduction.-The scattering matrix, or $S$ matrix, is undoubtedly one of the most important observables in particle as well as gravitational physics, relating asymptotic states at past and future infinity. It has long been known that its general properties, unitarity, analyticity, and crossing symmetry, imply dispersion relations between forward elastic scattering amplitudes (i.e., at vanishing scattering angle) and total cross sections. Such relations in turn yield positivity bounds for amplitudes evaluated in the infrared (IR), those measured in experiments. Dispersion relations therefore provide nontrivial constraints on the operators' coefficients in the effective field theories (EFTs) that are used to calculate the amplitudes at low energy $[1,2]$. An EFT with operators entering the action with the "wrong" sign cannot arise as the low-energy limit of a consistent ultraviolet (UV) theory satisfying the $S$-matrix axioms, and thus it lives in the "swampland." The proof of the $a$ theorem $[3,4]$ is perhaps the prime example of an application of these positivity bounds.

In this Letter, new amplitudes' positivities are derived for theories with a massless graviton in the spectrum, despite the fact that the forward elastic 2-to-2 scattering is universally singular due to graviton exchange in the $t$ channel. The new positivity bounds, and the way we circumvent the graviton forward singularity, are extremely important because they allow us to address the swampland program of quantum gravity and modified gravity theories, providing general and robust results.

Published by the American Physical Society under the terms of the Creative Commons Attribution 4.0 International license. Further distribution of this work must maintain attribution to the author(s) and the published article's title, journal citation, and DOI. Funded by SCOAP.
As a notable application, we study the Einstein-Maxwell theory, the low-energy EFT of an Abelian $U(1)$ gauge theory coupled to gravity, and show that our positivity bounds imply certain inequalities among its leading higherdimensional operators. Such operators are particularly relevant in the context of black hole physics-they affect the black hole's extremality condition, the minimal mass for which a charged black hole can exist. Our bounds imply that extremal black holes of mass $M$ and $U(1)$ charge $Q$ must satisfy $\sqrt{2} m_{\mathrm{Pl}}|Q| / M>1$; thus they are self-repulsive and no longer kinematically forbidden from decaying into smaller extremal black holes. This result constitutes a proof of (the mild form of) the celebrated weak gravity conjecture (WGC) [5]: extremal black holes are themselves charged states in the theory for which gravity is the weakest force.

Another interesting application of our bounds is on weakly broken Galileons, hypothetical scalars whose special properties make them very interesting candidates for a modified theory of gravity. We show, however, that these states have in fact a tiny cutoff if they are to originate from a canonical microscopic $S$ matrix, $\Lambda_{\mathrm{UV}}<$ a few $\times$ $\left(H^{3} m_{\mathrm{Pl}}\right)^{1 / 4} \sim 1 /\left(10^{7} \mathrm{~km}\right)$; i.e., their EFT breaks down at energies orders of magnitude smaller than the scale characterizing their interactions, the so-called strong coupling scale $\Lambda_{3}=\left(H^{2} m_{\mathrm{Pl}}\right)^{1 / 3} \sim 1 /\left(10^{3} \mathrm{~km}\right)$. Finally, we comment on how the EFTs of axions and $P(X)$ theories, of relevance in cosmology, are also subject to our constraints.

Regulating the forward limit.-The forward elastic amplitude of massless particles of polarizations $z_{i}$ is dominated by the universal Coulomb singularity

$$
\mathcal{M}^{z_{1} z_{2}}(s, t \rightarrow 0)=-\frac{s^{2}}{m_{\mathrm{Pl}}^{2} t}+O(s)
$$


because of the equivalence principle or, equivalently, because of factorization of the amplitude at the pole into the soft emission of an on-shell massless graviton, which has universal strength given by the reduced Planck mass $m_{\mathrm{Pl}}$. Since the coefficient of $s^{2}$ in Eq. (1) would enter the dispersion relation in the forward limit for particles of any spin $z_{i}$ [2], see Eq. (3), the naive application of the Cauchy integral theorem to $\mathcal{M}^{z_{1} z_{2}}(s, t \rightarrow 0)$ yields $\infty=\infty$, which is consistent, but admittedly not very informative. However, this divergence is due to long-distance physics, i.e., vanishing exchanged momentum, corresponding to the graviton probing arbitrarily large macroscopic distances even for large center-of-mass energy squared $s$. In any EFT the presumption is that the IR physics is known; therefore, one should be able to track and resolve the source of the singularity. Indeed, we show below how to massage the dispersion relation into an effective, regulated expression $\infty-\infty=$ finite $>0$, returning something meaningful, free of ambiguity and in fact of a definite sign, which can be used for charting the swampland in gravitational theories.

The key observation is that the Coulomb singularity is due to the infinite flat-space volume: we regulate it by putting the system on a cylinder. We compactify one spatial direction on a circle of length $L$, while the other three spacetime dimensions remain flat and infinite. In this way we can still scatter 3D asymptotic states while at the same time getting rid of the Coulomb singularity. Indeed, there is no propagating massless graviton in $D=3$, and hence no $s^{2} / t$ term for any finite value of $L[6]$. The $4 \mathrm{D}$ graviton has not fully disappeared though - rather it has left three propagating avatars: $\hat{g}_{M N} \rightarrow\left\{\sigma, V_{\mu}\right.$, KK modes $\}$, where $\sigma$ is a massless dilaton, $V_{\mu}$ a massless (Abelian) graviphoton, and the Kaluza-Klein (KK) modes have masses $m_{n}^{2} \sim n^{2} / L^{2}$. In the limit $L \rightarrow \infty$, which we take at the end after isolating the diverging terms, one recovers the 4D dynamics we are interested in.

In the following we will be interested in scattering nongravitational massless states, for example, the 3D photon $A_{\mu}$ and the 3D scalar $\Phi$ that live inside the 4D photon $\hat{A}_{M}$, in the 4D Einstein-Maxwell theory reduced to 3D. Since there is no 3D massless graviton and the states are either gapped, nonpropagating, or equivalent to simple scalars, we require a 3D Froissart-like bound

$$
\lim _{s \rightarrow \infty}\left|\mathcal{M}^{z_{1} z_{2}}(s, t=0) / s^{2}\right| \rightarrow 0, \quad z_{i}=\Phi, A,
$$

where with a slight abuse of notation we are now using $z_{i}$ to label the scattered 3D states. This is just the same assumption of polynomial boundedness that one accepts in $4 \mathrm{D}$ to derive dispersion relations and positivity bounds for the coefficient of, e.g., $(\partial \pi)^{4}$ or $\left(F_{\mu \nu} F^{\mu \nu}\right)^{2}$, when 4D gravity is neglected or nondynamical $[1,15]$.

Therefore, under exactly the usual assumptions that lead to the familiar positivity bounds for systems of spin- 0 and spin-1 massless particles in 4D, and repeating similar steps to those outlined in, for example, Ref. [2], we obtain a (provisional) dispersion relation for our IR-regulated 4D gravitational theory

$$
a^{z_{1} z_{2}}=\frac{2}{\pi} \int_{0}^{\infty} \frac{d s}{s^{3}} \operatorname{Im} \mathcal{M}^{z_{1} z_{2}}(s, t=0)>0,
$$

where the low-energy scattering amplitude for the 3D states $z_{i}$ is now regular in the forward elastic limit

$$
\mathcal{M}^{z_{1} z_{2}}(s, t \rightarrow 0)=a^{z_{1} z_{2}} s^{2}+\cdots .
$$

The dimensional reduction to 3D has left a universal contribution from gravitational zero and KK modes. Each KK mode gives

$$
a_{\mathrm{KK}}^{z_{1} z_{2}} \propto \frac{1}{L^{2} m_{\mathrm{Pl}}^{4} m_{\mathrm{KK}}} \propto \frac{1}{L m_{\mathrm{Pl}}^{4}|n|},
$$

where we used the fact that the $n$th KK-mode mass is $m_{\mathrm{KK}} \propto|n| \pi / L$. While each such contribution is subleading with respect to the terms that we want to bound in the following sections, their sum is actually logarithmically divergent. In addition, zero-mode loops generate $s^{3 / 2}$ terms in the amplitude, which dominate over the $s^{2}$ terms at low energy, seemingly swamping again the information about $a^{z_{1} z_{2}}$. In fact, these problems can be easily solved because the right-hand side of the dispersion relation (3) reproduces the same growth, so these otherwise large terms cancel out between the two sides of Eq. (3). Indeed, since the integrand itself in Eq. (3) is positive by the optical theorem, schematically $\operatorname{Im} \mathcal{M}^{z_{1} z_{2}}(s, t=0)=\sum_{x}\left|\mathcal{M}^{z_{1} z_{2} \rightarrow x}\right|^{2} \times($ phasespace $)$, we can move to the left-hand side any contribution from intermediate states $x$ in $\left|\mathcal{M}^{z_{1} z_{2} \rightarrow x}\right|^{2}$ and still get a positivity bound due to the remaining set of intermediate states. Specifically, we can move to the left-hand side the contributions from the intermediate IR states, such as the KK modes or anything that is calculable within the EFT (e.g., IR loops, that is, the light multiparticle intermediate states). The zero- and KK-mode contributions get subtracted and one is left to calculate just the contact terms suppressed by the cutoff $\Lambda_{\mathrm{UV}}$, that is, those that are generated by integrating out genuine UV states.

Just to illustrate this general point with a simple treelevel example, let us consider $\Phi \Phi \rightarrow \Phi \Phi$ scattering with the exchange of a scalar state $S$ coupled to $(\partial \Phi)^{2}$,

$$
\mathcal{M}_{S}^{\Phi \Phi}(s, t)=-\frac{2 c}{m_{\mathrm{Pl}}^{2} L}\left(\frac{s^{2}}{s-m_{S}^{2}+i \epsilon}+\text { crossing }\right),
$$

where $c$ is a fixed $O(1)$ number. This contributes to $a^{z_{1} z_{2}}$ in Eq. (4) by an amount $a_{S}^{\Phi \Phi}=4 c /\left(m_{\mathrm{Pl}}^{2} L m_{S}^{2}\right)$. The imaginary part (associated with the production of $S$ ) is 


$$
\operatorname{Im} \mathcal{M}_{S}^{\Phi \Phi}(s, t=0)=\frac{2 \pi c}{m_{\mathrm{Pl}}^{2} L} m_{S}^{4} \delta\left(s-m_{S}^{2}\right)+\cdots,
$$

precisely such that

$$
a_{S}^{\Phi \Phi}-\frac{2}{\pi} \int_{0}^{\infty} \frac{d s}{s^{3}} \operatorname{Im} \mathcal{M}_{S}^{\Phi \Phi}(s, t=0)=0,
$$

as expected on general grounds.

The KK-mode contributions to $a^{z_{1} z_{2}}$ in Eq. (5) actually arise at one loop, but the reasoning based on the optical theorem is completely general and works as in the previous example. This can be understood by discretizing the KK branch cut in a series of poles. Likewise for the contribution of the zero modes. The concrete details of how these contributions are subtracted are given in the Supplemental Material [7]. Here we note only that the KK modes, which grow the "extra" dimension as seen from a low-energy 3D observer, reproduce nicely the 4D universal gravitational contribution to the renormalization group running of $a^{z_{1} z_{2}}$. Since we can subtract it, which amounts to setting the renormalization scale at which $a^{z_{1} z_{2}}$ is evaluated at the cutoff where UV and IR amplitudes are matched, our final dispersion relation properly captures the UV physics that we are interested in.

All in all, our provisional dispersion relation (3) is rearranged into a much more informative expression

$$
a^{z_{1} z_{2}}-a_{\mathrm{KK}, \mathrm{IR}}^{z_{1} z_{2}}=\frac{2}{\pi} \int_{0}^{\infty} \frac{d s}{s^{3}} \operatorname{Im} \tilde{\mathcal{M}}^{z_{1} z_{2}}(s, t=0)>0,
$$

where $\tilde{\mathcal{M}}$ is the amplitude with the aforementioned gravitational zero- and KK-mode loop contributions subtracted. The left-hand side is therefore obtained by taking into account only the $s^{2}$ contributions to the elastic $z_{1} z_{2}$ scattering due to the tree-level interactions with massless particles such as the graviphoton and the dilaton, as well as the UV generated contact terms. The two sides (factor $L^{-1}$ ) of the subtracted dispersion relation (9) are not only finite for $L \rightarrow \infty$ but also positive because of the optical theorem. We note that removing the IR modes from the positivity bound is always possible but is useful in practice only for $\mathrm{UV}$ completions that are not strongly coupled at $\Lambda_{\mathrm{UV}}$, because it would become murky to assign what is IR (KK) and what is UV physics around the scale $\Lambda_{\mathrm{UV}}$. The subtracted dispersion relation is instead sharp and useful for weakly coupled UV completions.

Einstein-Maxwell EFT.-Let us focus on the important example of the Einstein-Maxwell EFT, whose leading 4D operators are

$$
\begin{aligned}
S= & \int d^{4} x \sqrt{|\hat{g}|}\left[\frac{m_{\mathrm{Pl}}^{2}}{2} \hat{R}-\frac{1}{4} \hat{F}^{M N} \hat{F}_{M N}+\frac{\alpha_{1}}{4 m_{\mathrm{Pl}}^{4}}\left(\hat{F}^{M N} \hat{F}_{M N}\right)^{2}\right. \\
& \left.+\frac{\alpha_{2}}{4 m_{\mathrm{Pl}}^{4}}\left(\hat{\widetilde{F}}^{M N} \hat{F}_{M N}\right)^{2}+\frac{\alpha_{3}}{2 m_{\mathrm{Pl}}^{2}} \hat{F}_{A B} \hat{F}_{C D} \hat{W}^{A B C D}\right],
\end{aligned}
$$

where $\hat{W}^{A B C D}$ is the Weyl tensor and $\hat{\widetilde{F}}_{M N}=\epsilon_{M N A B} \hat{F}^{A B} / 2$. The dependence on the UV scale $\Lambda_{\mathrm{UV}}$ that generates the $\alpha_{i}$ is absorbed into their definitions. These are the most general (parity preserving) four-derivative operators, up to field redefinitions $[17,18]$. In order to regulate the 4D forward limit and apply the positivity bounds (9), we compactify the $z$ direction as described in the previous section,

$$
\begin{gathered}
d \hat{s}_{4}^{2}\left[\hat{g}_{M N}\right]=e^{\sigma} d s_{3}^{2}\left[g_{\mu \nu}\right]+e^{-\sigma}\left(d z+V_{\mu} d x^{\mu}\right)^{2}, \\
\hat{A}_{M} d x^{M}=A_{\mu} d x^{\mu}+\Phi d z
\end{gathered}
$$

where all of the 3D fields are functions only of $(t, x, y)$. Focusing on terms which contribute to the $s^{2}$ part of the amplitude for $\Phi \Phi \rightarrow \Phi \Phi, A A \rightarrow A A$, and $\Phi A \rightarrow \Phi A$ only, the terms in the action that we must retain are

$$
\begin{aligned}
S= & L \int d^{3} x \sqrt{-g}\left\{\frac{m_{\mathrm{Pl}}^{2}}{2}\left(R-\frac{1}{2}(\partial \sigma)^{2}-\frac{1}{4} V^{2}\right)-\frac{1}{4}(1-\sigma) F^{2}-\frac{1}{2}(1+\sigma)(\partial \Phi)^{2}-\frac{1}{2} F_{\mu \nu} V^{\mu \nu} \Phi\right. \\
& +\frac{\alpha_{1}}{4 m_{\mathrm{Pl}}^{4}}\left(F^{2}+2(\partial \Phi)^{2}\right)^{2}+\frac{\alpha_{2}}{m_{\mathrm{Pl}}^{4}}\left(\epsilon^{\mu \nu \rho} F_{\mu \nu} \partial_{\rho} \Phi\right)^{2}+\frac{\alpha_{3}}{m_{\mathrm{Pl}}^{4}}\left[F_{\rho \mu} F^{\rho \nu} F^{\mu \sigma} F_{\nu \sigma}-\frac{1}{2} F^{4}-(\partial \Phi)^{4}+\frac{1}{2} F^{2}(\partial \Phi)^{2}\right] \\
& \left.-\frac{\alpha_{3}}{m_{\mathrm{Pl}}^{2}}\left(F_{\rho \mu} F_{\nu}^{\rho}-\partial_{\mu} \Phi \partial_{\nu} \Phi\right) \nabla^{\mu} \nabla^{\nu} \sigma-\frac{\alpha_{3}}{m_{\mathrm{Pl}}^{2}} F_{\mu \nu} \partial_{\rho} \Phi\left(\nabla^{\rho} V^{\mu \nu}+g^{\mu \rho} \nabla_{\alpha} V^{\nu \alpha}\right)\right\},
\end{aligned}
$$

where $F^{2}=F_{\mu \nu} F^{\mu \nu}$, and the same holds for $V$. To arrive at this expression, we made a field redefinition $A_{\mu} \rightarrow A_{\mu}+\Phi V_{\mu}$ to make gauge invariance manifest. Since the $g_{\mu \nu}$ propagates no degrees of freedom in $D=3$, we integrated it out, which is effectively equivalent to plugging the lowest-order equations of motion
$R^{\mu \nu}-\frac{1}{2} g^{\mu \nu} R=T^{\mu \nu} /\left(L m_{\mathrm{Pl}}^{2}\right) \quad$ into the interaction terms, generating new contact terms. Finally, we made a further field redefinition to remove interaction terms with $\square \sigma$.

The associated subtracted forward elastic scattering amplitudes are 


$$
\begin{gathered}
\tilde{\mathcal{M}}(\Phi \Phi \rightarrow \Phi \Phi)(s, t=0)=\frac{2 s^{2}}{m_{\mathrm{Pl}}^{4} L}\left(2 \alpha_{1}-\alpha_{3}\right)>0, \\
\tilde{\mathcal{M}}(A A \rightarrow A A)(s, t=0)=\frac{2 s^{2}}{m_{\mathrm{Pl}}^{4} L}\left(2 \alpha_{1}+\alpha_{3}\right)>0, \\
\tilde{\mathcal{M}}(\Phi A \rightarrow \Phi A)(s, t=0)=\frac{4 s^{2}}{m_{\mathrm{Pl}}^{4} L} \alpha_{2}>0 .
\end{gathered}
$$

Therefore, the associated positivity bounds read

$$
\begin{gathered}
2 \alpha_{1}-\alpha_{3}>0, \\
2 \alpha_{1}+\alpha_{3}>0, \\
\alpha_{2}>0,
\end{gathered}
$$

or, equivalently, $\alpha_{1}>\left|\alpha_{3}\right| / 2, \alpha_{2}>0$. These new positivity bounds are one of the main results of this Letter.

Remarkably, these bounds are stronger, meaning more general, than just a pure 4D Euler-Heisenberg EFT without gravity $[1,2,19]$, and they carry extra information about $\alpha_{3}$, which enters the black hole extremality condition, as we discuss in the next section.

Moreover, our homogeneous bounds (17)-(19) are distinct from the order-of-magnitude causality bounds on $O\left(\left|\alpha_{3}\right|\right) \quad[19,20]$, which are derived while assuming positivity of time delay and tree-level UV completion of the Einstein-Maxwell Lagrangian. See also Refs. [20,21] for a nice discussion of detectability of superluminal propagation within an EFT, and how the Euler-Heisenberg Lagrangian limit of real-world QED avoids superluminality [22].

It is interesting to compare the bounds (17)-(19) with the 4D calculation of the same processes retaining the Coulomb singularity in the $t \rightarrow 0$ limit,

$$
\begin{gathered}
\mathcal{M}_{4 \mathrm{D}}^{\downarrow \downarrow}=-\frac{s^{2}}{m_{\mathrm{Pl}}^{2} t}-\frac{s}{m_{\mathrm{Pl}}^{2}}+\frac{2 s^{2}\left(2 \alpha_{1}-\alpha_{3}\right)}{m_{\mathrm{Pl}}^{4}}, \\
\mathcal{M}_{4 \mathrm{D}}^{\uparrow \uparrow}=-\frac{s^{2}}{m_{\mathrm{Pl}}^{2} t}-\frac{s}{m_{\mathrm{Pl}}^{2}}+\frac{2 s^{2}\left(2 \alpha_{1}+\alpha_{3}\right)}{m_{\mathrm{Pl}}^{4}}, \\
\mathcal{M}_{4 \mathrm{D}}^{\uparrow \downarrow}=-\frac{s^{2}}{m_{\mathrm{Pl}}^{2} t}-\frac{s}{m_{\mathrm{Pl}}^{2}}+\frac{4 s^{2} \alpha_{2}}{m_{\mathrm{Pl}}^{4}},
\end{gathered}
$$

where the up and down arrows represent the two choices of real linear polarizations (corresponding to crossing symmetric amplitudes [2,23]). The lesson is that our 4Dregulated calculation, which works with 3D Lorentz invariance of the cylinder, teaches us which finite parts we are allowed to retain for the positivity bounds: throw away the $s^{2} / t$ singularity, the finite $O(s)$ term, but retain precisely the $O\left(s^{2}\right)$ term.
This immediately prompts us to expect a continuous set of positivity bounds associated with arbitrary linear polarizations $\left|c_{1,2}\right\rangle=\left(c_{\theta_{1,2}}\left|\uparrow_{1,2}\right\rangle+s_{\theta_{1,2}}\left|\downarrow_{1,2}\right\rangle\right)$, namely,

$$
\alpha_{3}\left(c_{2 \theta_{1}}+c_{2 \theta_{2}}\right)+4 \alpha_{1} c_{\theta_{1}+\theta_{2}}^{2}+4 \alpha_{2} s_{\theta_{1}+\theta_{2}}^{2}>0,
$$

where $c_{\theta}=\cos \theta$ and $s_{\theta}=\sin \theta$.

WGC and extremal black holes.-The leading higher-dimensional corrections $\alpha_{i}$ in the 4D EinsteinMaxwell EFT (10) modify the black hole extremality condition to [24]

$$
\left(\frac{\sqrt{2}|Q|}{M / m_{\mathrm{Pl}}}\right)_{\mathrm{extr}}=1+\frac{4}{5} \frac{(4 \pi)^{2} m_{\mathrm{Pl}}^{2}}{M^{2}}\left(2 \alpha_{1}-\alpha_{3}\right)>1,
$$

where $M$ is the black hole mass and $Q$ its charge (including the gauge coupling), and we work around $M \simeq Q m_{\mathrm{PI}} \sqrt{2}$. Remarkably, on the right-hand side of this expression, one finds the same combination, $2 \alpha_{1}-\alpha_{3}$, bounded to be positive by Eq. (17). Therefore, positivity bounds imply a greater charge-to-mass ratio for extremal black holes than in pure general relativity coupled minimally to an Abelian $U(1)$ gauge theory. The lighter the extremal black hole, the larger the charge-to-mass ratio. Extremal black holes within the validity of the 4D EFT, i.e., whose Schwarzschild radius $r_{s}=M / 4 \pi m_{\mathrm{Pl}}^{2}$ is larger than $1 / \Lambda_{\mathrm{UV}}$, are therefore selfrepulsive.

The positivity bound (17) implies the mild form of the WGC [5], which posits that a consistent theory of quantum gravity must contain massive charged states in the spectrum with $|q|>m /\left(\sqrt{2} m_{\mathrm{Pl}}\right)$ : the extremal black holes of Eq. (24) are such states. As a result, the paradox of stable extremal black holes has evaporated since extremal black holes are no longer kinematically forbidden to decay. Indeed, an extremal black hole of mass $M$ and charge $Q$ cannot decay into states that all have larger mass-to-charge ratio since the spectrum of masses and charges $\left(m_{i}, q_{i}\right)$ is constrained by $M>\sum_{i} m_{i}$ and $Q=\sum_{i} q_{i}$, whereas $\sum_{i} m_{i}=\sum_{i}\left|q_{i}\right| m_{i} /\left|q_{i}\right|>M$, which would be a contradiction. This argument is evaded precisely by decay products that contain one smaller extremal black hole, which has smaller mass-to-charge ratio (24) because of the positivity bound (17).

Since the same combination of EFT coefficients, $2 \alpha_{1}-\alpha_{3}$, enters the Wald entropy shift [18,19], our positivity bound (17) implies a larger black hole entropy as well. (Positivity bounds on even higher-derivative terms [23] imply a positive shift of the Kerr black hole entropy [25].)

Bounds on Galileons, axions, and $P(X)$.- Our IRregulated positivity bounds can now be used to constrain other interesting theories that have a massless graviton in the spectrum, e.g., those that are formulated in the context of modified gravity such as the weakly broken Galileons $[26,27]$ 
$\mathcal{L}=-\frac{1}{2}(\partial \pi)^{2}-\frac{1}{2 \Lambda_{3}^{3}}(\partial \pi)^{2} \square \pi+\frac{1}{4 \Lambda_{2}^{4}}(\partial \pi)^{4}+\cdots$,

where one can imagine the natural situation where $\Lambda_{2} \gg \Lambda_{3}$ since $(\partial \pi)^{4}$ weakly breaks the Galilean symmetry, whereas $(\partial \pi)^{2} \square \pi$ is an invariant. It was shown indeed that the hierarchy

$$
\Lambda_{2}^{4} \simeq H^{2} m_{\mathrm{Pl}}^{2}, \quad \Lambda_{3}^{3} \simeq H^{2} m_{\mathrm{Pl}},
$$

where $H$ is the Hubble constant, is stable under the loop corrections due to gravity that break the Galileon symmetry [27].

However, as estimated in Refs. [2,28] and calculated in detail in Ref. [29], scales $\Lambda_{2}$ and $\Lambda_{3}$ cannot be arbitrarily separated while keeping the cutoff $\Lambda_{\mathrm{UV}}$ fixed in a theory without gravity, parametrically $1 / \Lambda_{2}^{4}>\Lambda_{\mathrm{UV}}^{8} / \Lambda_{3}^{12}$. We can now see that a similar bound survives even when the graviton is dynamical if the UV completion is assumed to be weakly coupled (at least up to $\Lambda_{2}$ ). Following the arguments of the previous sections, we can subtract the gravitational $\mathrm{KK}$ modes after the 3D compactification, and we then extract the following bound,

$$
\frac{1}{\Lambda_{2}^{4} L}>\frac{2}{\pi} \int \frac{\Lambda_{\mathrm{UV}}^{2}}{s^{3}} \operatorname{Im} \tilde{\mathcal{M}}^{\pi \pi}(s)>\frac{c}{16 \pi^{2}} \frac{\Lambda_{\mathrm{UV}}^{8}}{L \Lambda_{3}^{12}},
$$

where we used the positivity of the integrand in Eq. (9) and retained from the optical theorem only the inelastic cross section into pairs of Galileon KK modes $\pi_{k}$, $\sum_{k, m_{k}<\Lambda_{\mathrm{UV}}} \sigma\left(\pi \pi \rightarrow \pi_{k} \pi_{k}\right)$. The constant $c=O\left(10^{-4}\right)$ is an inessential numerical factor resulting from integrating over the phase space and then along the branch cut. The bound (27) nicely reproduces the scaling from the calculation without gravity [29]. As a consequence, hierarchy (26) between $\Lambda_{2}$ and $\Lambda_{3}$, which is stable because of symmetry, in fact requires an extremely small cutoff,

$$
\Lambda_{\mathrm{UV}}<\left(H^{3} m_{\mathrm{Pl}}\right)^{1 / 4}\left(\frac{16 \pi^{2}}{c}\right)^{1 / 8} \sim \frac{1}{10^{7} \mathrm{~km}},
$$

in order to be consistent with the beyond positivity bound (27) that applies in a gravity theory.

We can also consider

$$
\mathcal{L}=-\frac{1}{2}(\partial \phi)^{2}+\frac{a}{4 f^{4}}(\partial \phi)^{4}+\cdots,
$$

describing axions, or $P(X)$ theories with $X=(\partial \phi)^{2}$ and $f \sim \Lambda_{2}$ in Eq. (26). Our positivity bounds imply that $a>0$, even in the presence of gravity-such a constraint was not possible before because of the inherent long-distance sensitivity, $t \sim H^{2}$, of their forward scattering amplitudes.

Conclusions. - In this Letter we derived new amplitudes' positivities in quantum gravity in four dimensions. We showed how to regulate and subtract the gravitational
Coulomb singularity in the forward elastic limit by putting the theory on a cylinder, using its 3D scattering states and then restoring $4 \mathrm{D}$ spacetime. This method allowed us to extract positivity bounds on the $s^{2}$ coefficient of the EFT amplitudes removing the $t$-channel graviton singularity in a controlled way. Remarkably, the resulting positivity bounds are generically different than those obtained in flat space without gravity. This is due to the contribution to the amplitudes from the dilaton and the graviphoton (on top of the contact terms from the nondynamical metric), which remain dynamical even on the cylinder and leave their finite gravitational footprint in the 4D limit.

As an important application, we studied the EinsteinMaxwell EFT and showed that the positivity bounds imply that extremal black holes have a charge-to-mass ratio larger than 1, which is approached from above as the mass is increased. This provides an $S$-matrix proof of the mild form of the WGC since it implies that extremal black holes are self-repulsive, $|Q|>M$ in suitable units. The amplitudes' positivity implies as well that the Wald entropy shift due to the leading higher-dimensional operators is always positive.

In the context of the swampland program, these are perhaps somewhat negative results since they lower the expectations that the WGC is useful for charting the landscape of consistent theories of quantum gravity. Any weakly coupled UV completion with a canonical $S$ matrix gives rise to unstable extremal black holes. Of course, it may be that string theory is the only such UV completion.

We considered other important applications in cosmology within the context of modified gravity. In particular, we found that perturbative UV completions of Galileons can be consistent with the (beyond) positivity bounds in a theory with a massless graviton only if the cutoff of the theory is at least as small as a few $\times\left(H^{3} m_{\mathrm{Pl}}\right)^{1 / 4}$.

This work opens the door to a future understanding of other quantum gravity conjectures in terms of amplitudes' positivity.

We would like to thank Luca Martucci, Riccardo Rattazzi, Francesco Riva, Marco Serone, Francesco Sgarlata, and Filippo Vernizzi for the useful discussions. We also thank Miguel Montero for the interesting comments, and Garret Goon for the discussions on the scattering of gravitational states. We are especially grateful to Toshifumi Noumi, Clifford Cheung, and Grant Remmen for the discussions on the soft limit. B. B. thanks Sergei Dubovsky for the lively discussions about the existence of analytic scattering amplitudes in 3D conical spaces. J. S. is supported by the Collaborative Research Center SFB1258 and the DFG cluster of excellence EXC 153, "Origin and Structure of the Universe.” M. L. acknowledges financial support from the Enhanced Eurotalents fellowship, a Marie Skłodowska-Curie Actions program, and the European Research Council under Grant No. ERC-STG-639729, "preQFT: Strategic Predictions for Quantum Field Theories." 
[1] A. Adams, N. Arkani-Hamed, S. Dubovsky, A. Nicolis, and R. Rattazzi, Causality, analyticity and an IR obstruction to UV completion, J. High Energy Phys. 10 (2006) 014.

[2] B. Bellazzini, Softness and amplitudes positivity for spinning particles, J. High Energy Phys. 02 (2017) 034.

[3] Z. Komargodski and A. Schwimmer, On renormalization group flows in four dimensions, J. High Energy Phys. 12 (2011) 099.

[4] M. A. Luty, J. Polchinski, and R. Rattazzi, The $a$-theorem and the asymptotics of 4D quantum field theory, J. High Energy Phys. 01 (2013) 152.

[5] N. Arkani-Hamed, L. Motl, A. Nicolis, and C. Vafa, The string landscape, black holes and gravity as the weakest force, J. High Energy Phys. 06 (2007) 060.

[6] The tree-level exchange of a 3D auxiliary 2 tensor $g_{\mu \nu}$ does give rise to an $s^{2} / t$ term. However, since the associated singularity does not correspond to any physical particlei.e., there is no physical graviton in $D=3$-higher-order corrections actually remove it. In the Supplemental Material [7] we show that the 3D Einstein-Hilbert amplitude is indeed regular in the forward limit (an irrelevant constant in fact), see also [8-13]. This should be contrasted with the $D=4$ case, where no correction can erase the $t$-channel pole.

[7] See Supplemental Material at http://link.aps.org/ supplemental/10.1103/PhysRevLett.123.251103 for a detailed discussion of the contributions to the dispersion relation from the zero and KK modes (see also Ref. [14]), and of some of the features of 3D scattering amplitudes.

[8] M. Ciafaloni, Self-consistent scattering matrix in $(2+1)$ gravity, Phys. Lett. B 291, 241 (1992).

[9] G. 't Hooft, Nonperturbative two particle scattering amplitudes in $(2+1)$-dimensional quantum gravity, Commun. Math. Phys. 117, 685 (1988).

[10] S. Deser, J. G. McCarthy, and A. R. Steif, Ultra-Planck scattering in $D=3$ gravity theories, Nucl. Phys. B412, 305 (1994).

[11] M. Zeni, Forward scattering in $(2+1)$ quantum gravity, Classical Quantum Gravity 10, 905 (1993).

[12] D. N. Kabat and M. Ortiz, Eikonal quantum gravity and Planckian scattering, Nucl. Phys. B388, 570 (1992).

[13] G. 't Hooft, Graviton dominance in ultrahigh-energy scattering, Phys. Lett. B 198, 61 (1987).

[14] W.-M. Chen, Y.-t. Huang, T. Noumi, and C. Wen, Unitarity bounds on charged/neutral state mass ratio, Phys. Rev. D 100, 025016 (2019).

[15] In the Supplemental Material [7] we discuss how our conclusions adapt to relaxing the Froissart bound (2). We show in particular that an asymptotic form of the WGC, i.e., for very large extremal black holes, can still be proven even when we assume no Froissart-like bound; see also Ref. [16] for a direct calculation of the beta functions.

[16] S. Deser and P. van Nieuwenhuizen, One-loop divergences of quantized Einstein-Maxwell fields, Phys. Rev. D 10, 401 (1974).

[17] C. Cheung and G. N. Remmen, Infrared consistency and the weak gravity conjecture, J. High Energy Phys. 12 (2014) 087.

[18] C. Cheung, J. Liu, and G. N. Remmen, Proof of the weak gravity conjecture from black hole entropy, J. High Energy Phys. 10 (2018) 004.

[19] Y. Hamada, T. Noumi, and G. Shiu, Weak gravity conjecture from unitarity and causality, Phys. Rev. Lett. 123, 051601 (2019).

[20] X. O. Camanho, J. D. Edelstein, J. Maldacena, and A. Zhiboedov, Causality constraints on corrections to the graviton three-point coupling, J. High Energy Phys. 02 (2016) 020.

[21] G. Goon and K. Hinterbichler, Superluminality, black holes and EFT, J. High Energy Phys. 02 (2017) 134.

[22] I. T. Drummond and S. J. Hathrell, QED vacuum polarization in a background gravitational field and its effect on the velocity of photons, Phys. Rev. D 22, 343 (1980).

[23] B. Bellazzini, C. Cheung, and G. N. Remmen, Quantum gravity constraints from unitarity and analyticity, Phys. Rev. D 93, 064076 (2016).

[24] Y. Kats, L. Motl, and M. Padi, Higher-order corrections to mass-charge relation of extremal black holes, J. High Energy Phys. 12 (2007) 068.

[25] H. S. Reall and J. E. Santos, Higher derivative corrections to Kerr black hole thermodynamics, J. High Energy Phys. 04 (2019) 021.

[26] A. Nicolis, R. Rattazzi, and E. Trincherini, The Galileon as a local modification of gravity, Phys. Rev. D 79, 064036 (2009).

[27] D. Pirtskhalava, L. Santoni, E. Trincherini, and F. Vernizzi, Weakly broken Galileon symmetry, J. Cosmol. Astropart. Phys. 09 (2015) 007.

[28] A. Nicolis, R. Rattazzi, and E. Trincherini, Energy's and amplitudes' positivity, J. High Energy Phys. 05 (2010) 095; 11 (2011) 128.

[29] B. Bellazzini, F. Riva, J. Serra, and F. Sgarlata, Beyond Positivity Bounds and the Fate of Massive Gravity, Phys. Rev. Lett. 120, 161101 (2018). 